$>$ Objective: to investigate the safety and longterm efficacy of core decompression with synthetic calcium-sulfate bone grafting on non-traumatic osteonecrosis of the femoral head.

Method: a total of 76 patients with non-traumatic osteonecrosis of the femoral head were admitted to our hospital from February 2012 to November 2014 and included in the study. All the patients were treated by core decompression with synthetic calcium-sulfate bone grafting. After treatment, the patients were followed-up for 12 months. The operation time, intraoperative blood loss, and perioperative complications were also recorded intraoperatively to evaluate the clinical efficacy and safety. The preoperative and postoperative pain scores measured by visual analogy score (VAS) were also recorded and compared.

Results: all of the 76 patients were successfully operated and included in the study. The mean operation time was $34.6 \pm 14.2 \mathrm{~min}$, and the intraoperative blood loss was $23.6 \pm 8.9 \mathrm{~mL}$. The patients hip functions were assessed on the basis of the Harris hip score, 3 and 12 months after operation. The excellent rates 3 and 12 months after operation were significantly higher than those before operation $(P<0.05)$. The imaging stabilities of the hip joints 3 and 12 months after operation were significantly higher than those before operation $(P<0.05)$. The patient postoperative pain scores (VAS score) gradually decreased. The VAS scores 12 months after operation were significantly lower than those before operation $(P<0.05)$.

Conclusion: the long-term efficacy of core decompression with synthetic calcium-sulfate bone grafting on non-traumatic osteonecrosis of the femoral head is good and accompanied with significantly improved postoperative joint functions. < Key words: calcium sulfate; clinical efficacy; core decompression; femoral head; non-traumatic osteonecrosis.

\section{Analysis of \\ the long-term \\ efficacy of core \\ decompression \\ with synthetic \\ calcium-sulfate \\ bone grafting on \\ non-traumatic \\ osteonecrosis of \\ the femoral head}

Pengfei Zhao ${ }^{1}$, Jing $\mathrm{Hao}^{2}$

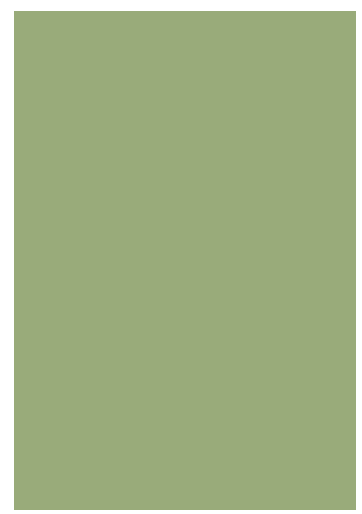

${ }^{1}$ Department of trauma orthopedics, $6^{\text {th }}$ affiliated hospital of Wenzhou medical university (Lishui People's Hospital), Lishui Zhejiang province, 323000 PR China. ${ }^{2}$ Department of general surgery, $6^{\text {th }}$ affiliated hospital of Wenzhou medical university (Lishui People's Hospital), Lishui Zhejiang province, No.15 Dazhong Road, Liandu district, Lishui 323000 PR China.

Corresponding author: Jing Hao jinghao1977@163.com

\section{Introduction}

The osteonecrosis of the femoral head is roughly divided into two types, namely, traumatic and non-traumatic [1, 2]. Nontraumatic osteonecrosis of the femoral head is mostly related to the long-term high-dose use of adrenocorticotropic hormones or a long history of heavy drinking; however, some patients with non-traumatic osteonecrosis of the femoral head have not experienced any of these habits. As such, they are usually diagnosed with spontaneous osteonecrosis of the femoral head, but the exact mechanism of this condition has yet to be clarified $[3,4]$. A consensus has been reached on the following therapeutic regimens against the early non-traumatic osteonecrosis of the femoral head: core decompression for patients in early stages and arthroplasty for patients in advanced stages [5]. In the present study, we have included 76 cases of non-traumatic osteonecrosis and evaluated the long-term efficacy of core decompression combined with synthetic calcium-sulfate bone grafting on nontraumatic osteonecrosis of the femoral head. Furthermore, we also have evaluated the pain scores and the rate of joint recovery 12 months after operation. 


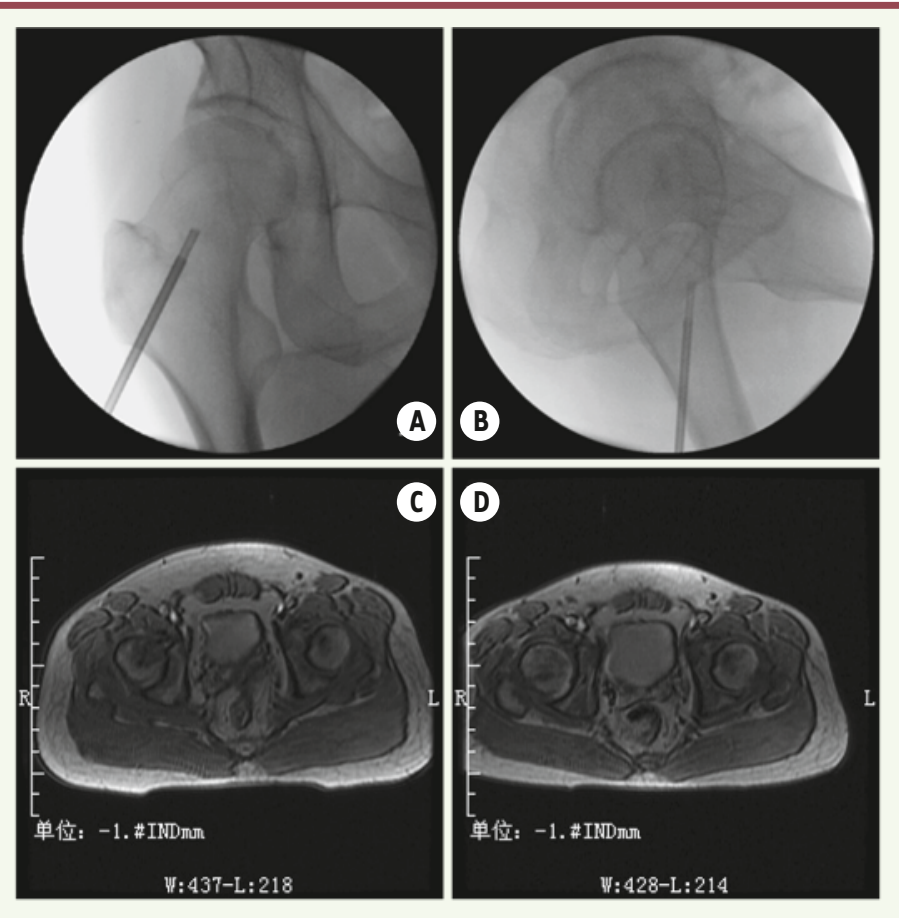

Figure 1. Bone marrow core decompression and calcium sulfate artificial bone implantation of pre-operative and post-operative imaging comparison. $A$ : X-ray below a hollow drill; a hollow drill in B: X-ray side; $C$ : double hip MRI before operation; $D$ : double hip MRI after operation.

\section{Materials and methods}

\section{Patients}

A total of 76 patients with non-traumatic osteonecrosis of the femoral head of unilateral hip were admitted to our hospital from February 2012 to November 2014 and were included in this study. 48 were males and 28 were females, with an average age of $51.2 \pm 15.6$ years old. 33 suffered from left hip diseases and 43 manifested right hip diseases. The patients were divided into two groups on the basis of Ficat osteonecrosis score: 26 cases in stage I and 50 cases in stage II. Among these patients, 30 used hormones, 27 had a long history of heavy drinking, and 19 suffered from unknown causes. The patients were diagnosed in accordance with the 2012 Expert Consensus on the Diagnosis and Treatment Criteria for Adult Osteonecrosis of Femoral Head.

\section{Treatment}

The patients were instructed to lie flat and were given continuous epidural anesthesia. The surgical field was conventionally draped with a sterile surgical towel. Through C-arm fluoroscopy, a positioning guide pin was inserted $2 \mathrm{~cm}$ below the apex of the rotor running along the upper center of the femoral head until $0.5 \mathrm{~cm}$ below the femoral cartilage was reached. Through $\mathrm{X}$-ray lateral projection positioning techniques, the guide pin was located at the focal area. With the guide pin as the center, a $3 \mathrm{~cm}$ surgical incision was made. A tunnel incision was also cut with a core drill running along the guide pin until the central area of the lesion was reached. The patients underwent lesion clearance through fluoroscopy. The synthetic bone graft of calcium sulfate composed of a paste mixed with powder and diluents was poured into the lesion zone by using a catheter connected to a pressure injector. After the lesions of the femoral head were completely filled, the catheter was drawn back step-by-step through fluoroscopy. The tunnel incision was filled with the remaining paste, the catheter was retreated to the cortical bone, the inner core of the catheter was plugged, and the catheter was pulled out until the implanted material was cured (Figure 1). Weight-bearing exercises of the affected limb were avoided for 6 weeks.

\section{Indexes for efficacy evaluation}

The patients were followed-up for 12 months after operation. The intraoperative blood loss and perioperative complications were recorded to assess the hip joint post-operative recovery rate and the imaging stability. The clinical efficacy was evaluated on the basis of the Harris hip score [6]: 90-100 points, excellent or hip joints without pain; 80-89 points, good or hip joints with mild discomfort and substantially unrestricted activities; 70-79 points, fair or hip joints with mild pain and slightly restricted activities; and below 70 points, poor. Imaging stability was evaluated on the basis of Ficat Osteonecrosis Score [7]: in stage I, X-ray reveals that the dead bone is replaced with a new bone and thus indicates significant recovery from necrosis; in Stage II, the X-ray shows that the new bone can support the load-bearing part, without any collapse or any aggravated collapse on the articular surface; in stage III, X-ray indicates that collapse or any aggravated collapse occurred on the articular surface and the condition exacerbates.

\section{Statistical analysis}

Data were analyzed using the SAS9.0 software. Measurement data were expressed as $\bar{x} \pm s$. Betweengroup comparison was conducted using a $t$-test. The counted data were expressed in terms of rate. Pre-/ post-operative comparison was performed via a $\chi^{2}$ test or Fisher's exact probability test. Differences were considered as significant when $P<0.05$.

\section{Results}

\section{Operation results}

All of the 76 patients were successfully operated. Peri-operative deaths or serious complications did not occur. The mean operation time was $34.6 \pm 14.2 \mathrm{~min}$ and the intraoperative blood loss was $23.6 \pm 8.9 \mathrm{~mL}$. 


\begin{tabular}{lccccc}
\hline Time & Excellent & Good & Fair & Bad & Excellent rate \\
\hline Pre-operation & $0(0.00)$ & $5(6.57)$ & $28(36.84)$ & $43(56.58)$ & $5(6.57)$ \\
\hline 3-month post-operation & $23(30.26)$ & $29(38.16)$ & $18(23.68)$ & $6(7.89)$ & $52(68.42) *$ \\
\hline 12-month post-operation & $30(39.47)$ & $31(40.79)$ & $12(15.79)$ & $3(3.95)$ & $61(80.26) *$ \\
\hline
\end{tabular}

Table 1. Postoperative excellent rates at different time points according Harris Hip score [n (\%)]. * Compared with pre-operation, $\mathrm{P}<0.05$.

\begin{tabular}{lcccc}
\hline Time & I & II & III & Stability rate \\
\hline Pre-operation & $0(0.00)$ & $10(13.16)$ & $66(86.84)$ & $10(13.16)$ \\
\hline 3-month post-operation & $24(31.58)$ & $25(32.49)$ & $27(35.53)$ & $49(64.47) \star$ \\
\hline 12-month post-operation & $62(81.58)$ & $5(6.58)$ & $9(11.84)$ & $67(88.16) \star$ \\
\hline
\end{tabular}

Table 2. Postoperative imaging stability at different time points [n (\%)]. * Compared with pre-operation, $\mathrm{P}<0.05$.

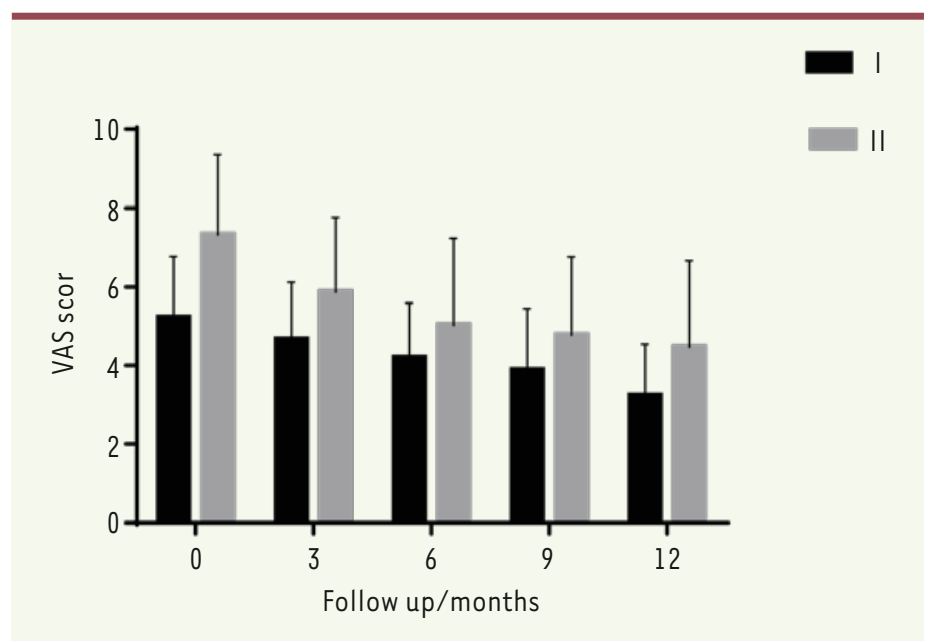

Figure 2. Post-operative VAS score comparison at different time points.

\section{Harris hip score}

The patient hip functions were assessed on the basis of Harris hip score, 3 and 12 months after operation. The excellent rates observed 3 and 12 months after operation were significantly higher than those before operation $(P<0.05$; Table 1$)$.

\section{Hip joint stability}

The patient hip joints were assessed based on Ficat osteonecrosis score, 3 and 12 months after operation. The imaging stabilities of the hip joints 3 and 12 months after operation were significantly higher than those before operation $(P<0.05$; Table 2$)$.

\section{Pain score comparison}

Twelve months after operation, the patient post-operative pain scores (VAS score) decreased gradually. Theses scores were significantly lower than before operation $(P<0.05)$, as shown in Figure 2.

\section{Discussion}

Osteonecrosis of the femoral head is a disease caused by osteocyte ischemia and necrosis, trabecular fracture, and femoral head collapse induced by a local blood circulation disorder of the femoral head because of various factors [8]. This disease is also caused by femoral head ischemia and osteocyte apoptosis as a consequence of blood circulation and bleeding disorders of the femoral head; this condition leads to trabecular fracture, subchondral fracture, and femoral head collapse [9]. The following therapeutic regimens have been generally used to treat early non-traumatic osteonecrosis of the femoral head: core decompression for patients in early stages and arthroplasty for patients in advanced stages [5]. Kong Guofan et al. [10] investigated the efficacy of percutaneous core decompression using bone grafting combined with interventional treatment on adult peri-collapse osteonecrosis of the femoral head. They concluded that this regimen can be used to effectively treat adult peri-collapse osteonecrosis of the femoral head with mini-invasion. This treatment procedure can also significantly relieve symptoms, improve hip functions, and prevent or retard femoral head collapse. Furthermore, the clinical efficacy of core decompression on early non-traumatic osteonecrosis of the femoral head has been confirmed [8]; However, whether this treatment is suitable for patients with non-traumatic osteonecrosis of the femoral head in stage II or above (Ficat) was unclear.

Previous studies have shown that the femoral head after simple decompression may not be supported 
immediately; as a consequence, the risk of femoral head collapse increases [11, 12]. The femoral head after core decompression combined with synthetic calcium-sulfate bone grafting may be supported, with a low risk of femoral head collapse. In osteogenesis for local bone defects, calcium sulfate hemihydrate powder forms a solid precipitate through an agglutination reaction after hydration; therefore, this substance can exhibit efficient bone conduction to establish a stable environment for the growth of new bone or can provide a strong mechanical support for regional grafting, bearing a portion of stress conduction [12, 13]. Under a fluoroscope, the synthetic bone graft of calcium sulfate is filled into the targeted lesion zone of the femoral head; as a result, the tissues of the necrotic area are replaced, and the synthetic bone graft of calcium sulfate acts as a scaffold conducive for the formation of new bone and provides a certain mechanical support before grafting [14].

In the present study, 76 patients with unilateral non-traumatic osteonecrosis of the femoral head were treated by core decompression with synthetic calcium sulfate bone grafting. The surgical procedure is simple, safe, and reliable. No serious perioperative complications were observed in the current study. The long-term efficacy of the treatment after operation was also assessed. Our results indicate that the excellent rate of the patient hip joints 12 months after operation is relatively high, with pain alleviated. Thus, the proposed strategy can be applied to treat non-traumatic osteonecrosis of the femoral head effectively. The method should be recommended for patients with early senile osteonecrosis of the femoral head. However, no control group was included in the study. It is an obvious limitation and it requires to develop further investigation. $\diamond$

\section{CONFLICT OF INTEREST}

The authors state that there have no conflict of interest.

\section{REFERENCES}

1. Mont MA, Cherian JJ, Sierra RJ, Jones LC, Lieberman JR. Nontraumatic osteonecrosis of the femoral head: where do we stand today? A ten-year update. J Bone Joint Surg Am 2015;97:1604-27.

2. Moya-Angeler J, Gianakos AL, Villa JC, Ni A, Lane JM. Current concepts on osteonecrosis of the femoral head. World J Orthop 2015;6:590-601.

3. Tripathy SK, Goyal T, Sen RK. Management of femoral head osteonecrosis: current concepts. Indian J Orthop 2015;49:28-45.

4. Plancher KD, Razi A. Management of osteonecrosis of the femoral head. Orthop Clin North Am 1997;28:461-77.

5. Zhao DW, Hu YC. Chinese experts' consensus on the diagnosis and treatment of osteonecrosis of the femoral head in adults. Orthop Surg 2012;4:125-30.

6. Bartonicek J, Vavra J, Bartoska R, Havranek P. Operative treatment of avascular necrosis of the femoral head after proximal femur fractures in adolescents. Int Orthop 2012;36:149-57.

7. Ficat RP. Aseptic necrosis of the femur head. Preliminary remarks concerning staging: stage 0. Acta orthopaedica Belgica 1981;47:239-41.

8. Zalavras CG, Lieberman JR. Osteonecrosis of the femoral head: evaluation and treatment. J Am Acad Orthop Surg 2014;22:455-64.

9. Issa K, Pivec R, Kapadia BH, Banerjee S, Mont MA. Osteonecrosis of the femoral head: the total hip replacement solution. Bone Joint J 2013;95B:46-50.

10. Kong FG, Han SH, Liu FW, Li YJ. Percutaneous core decompression combined with percutaneous coronary intervention for the treatment of femoral head necrosis in adults. Guide of China Medicine 2014;35: 24-26.

11. Musso ES, Mitchell SN, Schink-Ascani M, Bassett CA. Results of conservative management of osteonecrosis of the femoral head. A retrospective review. Clin Orthop Relat Res 1986:209-15.

12. Kamath AF, McGraw MH, Israelite CL. Surgical management of osteonecrosis of the femoral head in patients with sickle cell disease. World J Orthop 2015;6:776-82.

13. Samy AM. Management of osteonecrosis of the femoral head: a novel technique. Indian J Orthop 2016;50:359-65.

14. Guo KJ, Zhao FC, Guo Y, Li FL, Zhu L, Zheng W. The influence of age, gender and treatment with steroids on the incidence of osteonecrosis of the femoral head during the management of severe acute respiratory syndrome: a retrospective study. Bone Joint J 2014;96-B:259-62. 